\title{
Meteorological Application of UAV as a New Way of Vertical Profile of Lower Atmosphere Measurement
}

\author{
Josef NOVOTNÝ́ ${ }^{1}$, Radek BYSTřICKÝ ${ }^{2}$, Karel DEJMAL ${ }^{3}$ \\ 1,2,3 University of Defence in Brno, Faculty of Military Technology, Address: Kounicova.65, CZ- \\ 66210, Brno, The Czech Republic \\ E-mails: ${ }^{1}$ josef.novotny@unob.cz, ${ }^{2}$ radek.bystricky@unob.cz, ${ }^{3}$ karel.dejmal@unob.cz
}

\begin{abstract}
The knowledge of basic meteorological parameters is crucial in terms of understanding the atmospheric behavior for a given period and consequently also for creating the weather forecast. There are several ways of obtaining such data. A broadening of measurement techniques through the unmanned aerial vehicles (UAV) is one of the newest trends of today. Depending on the character of use of the unmanned platform, it could gather data from a significant part of low troposphere. Our experiences and problems with meteorological application of UAV is described in this paper.
\end{abstract}

KEY WORDS: unmanned aerial vehicles (UAV), aerological sounding, troposphere, meteorological parameters, accuracy of measurement

\section{Introduction}

The knowledge of basic meteorological parameters such as vertical profile of pressure, temperature, humidity and airflow speed and orientation, that describe the state of atmosphere in lower levels, is crucial in terms of understanding the atmospheric behavior for a given period and consequently also for creating the weather forecast [1]. There are several ways of obtaining such data. A majority of them, however, is in principle more complex and therefore more costly than measurements carried out on the ground within the terrestrial network of meteorological stations. In the past few years, the use of unmanned aerial vehicles (UAV) started to occur for other than camera related purposes such as identification of vegetation structure using LIDAR technology [2]. A broadening of measurement techniques through the aforementioned UAV is one of the newest trends of today [3]. This way of data acquisition could be classified as a local measuring technique and direct method. Depending on the character of use of the unmanned platform, it could gather data from a significant part of low troposphere [4], [5]. Our unmanned vehicle falls into category of controllable vehicles heavier than air with rotary wings with electrical engine weighting less than $7 \mathrm{~kg}$.

To be able to collect the meteorological information, the UAV should possess adequate integrable sensors. Those must be sufficiently sensitive and precise, but due to a limited loading capacity not too heavy. Despite having already given space to the comparison of different measurements [6],[7], the evaluation of drone measurement precision is much more complex, as the drone itself influences its environment, meteorological elements included (mixing of air, heat production). The sensors could be calibrated or tested [8], but making tests in the course of UAV's flight is quite difficult. Our evaluation of measurement usability focused on the comparison with other available data, whose quality however is not $100 \%$ known and thus may vary from the declared precision.

\section{Method of Investigation}

Drone measurements done at standstill were compared with data from meteorological station while measurements at flight faced comparison to baloon sounding and measuring using sensors attached to 250 meters high mast. Due to its structure, the UAV Robodrone SuperHornet (in Fig. 1) pertains to multi-engine vehicles (quadcopters) [9]. Besides the usual components, it also incorporates a telemetric module, suspension camera and meteorological data acquisition unit. Thanks to used engines and accumulators, the stamina of our vehicle amounts to 20-25 minutes, 10 minutes of which would be a safety reserve. For practical application, it is therefore counted with a flight of around 15 minutes. The concept of the vehicle allows flying under conditions such as high air humidity (rain) or a wind no greater than $20 \mathrm{~m} / \mathrm{s}$. Its vertical speed had been limited to $5 \mathrm{~m} / \mathrm{s}$, taking into account the capacity and discharge rates of accumulator. Bearing that capacity in mind, the flights could be, theoretically, conducted up until around $8200 \mathrm{ft}$ ( $2500 \mathrm{~m})$ above ground level (AGL). In respect of legislative limitations we can fly the vehicle in experimental mode in several designated areas up to $5000 \mathrm{ft}$ $(1500 \mathrm{~m})$ above mean sea level (AMSL) provided a reserved air space of $3 \mathrm{~km}$ radius.

The aim of the experiments is to approximate to the measuring conditions of meteorological balloon probe. The flights consist of a simple constant-speed ascent to the maximum height and back, while holding the same geographical coordinates. Even at the designing phase, it was clear, that the air turbulence around propellers would cause diversions in 
measured values, especially in the case of temperature. In order to stave off this negative effect a cheap meteorological data acquisition unit had to be installed. It serves to determine the impact on each given sensor and to find an appropriate location so that the impact in the course of flight is minimal. The key point in the development stage turned to be the choice of sensors with a very small time constant, as only the proximity of ground before the take-off can heat up or cool down the sensors by several degrees.

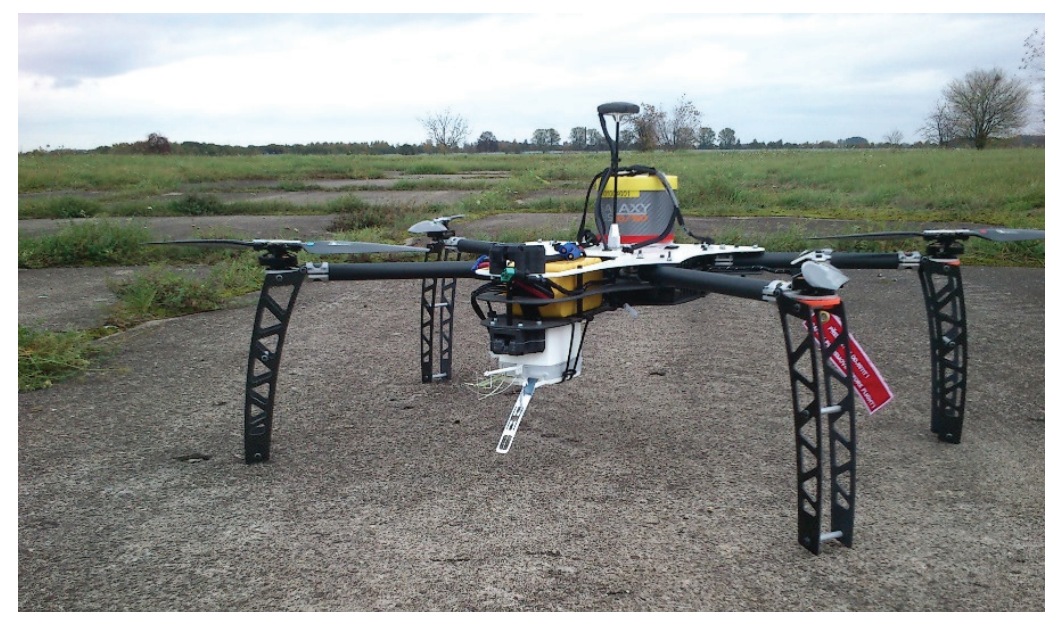

Fig. 1. UAV Robodrone SuperHornet

At present, the drone is fitted with pressure and humidity sensor BME280 by Bosch, which communicates via I2C interface. It measures humidity from $0 \%$ to $100 \%$ and pressure from $300 \mathrm{hPa}$ to $1100 \mathrm{hPa}$ with definition $0,008 \%$ for humidity and $0,18 \mathrm{~Pa}$ for pressure. The precision in the case of humidity is $\pm 3 \%$ and $\pm 1 \mathrm{~Pa}$ for the pressure. Additionally, it comprises a platinum temperature sensor 701-101BAA-B00 by Honeywell with measuring range of $-70{ }^{\circ} \mathrm{C}$ to $500{ }^{\circ} \mathrm{C}$ and definition that thanks to the used processing chain amounts to $0,001{ }^{\circ} \mathrm{C}$. At this point, the air direction and airspeed data are not usually available, but we try to obtain those experimentally through calculation from measured position angles. The preliminary results are quite promising.

\section{Results}

The initial verification of drone abilities took place in military barracks Černá Pole in Brno, when the UAV was at standstill. It was put inside a Stevenson screen and the values obtained were compared to those from automatic sensors of the meteorological station METEOS6. The first measurement showed that the operating drone battery (power for measuring as well as flying) even in a still position produces heat that overvalues the temperature. Better results came out when the battery was removed from the Stevenson screen and replaced only by cables. Nevertheless the data sometimes varied substantially (by several degrees). The main reason was probably radiation. The Stevenson screen was situated about $5 \mathrm{~m}$ from automatic sensors and in its proximity were objects (buildings, tress...) that casted odd shadows whether on sensors or box. The radiation had moreover better access to drone sensor that is not covered, where the bottom part of the Stevenson screen is constituted by a thick foundation that practically does not impede the penetration of reflected sun radiation. The sensors in automatic station are much better protected. Another possible cause for difference is different ventilation of both places. In order to eliminate those factors we aimed to execute the measurements at cloudy and windy weather conditions. The drone was in the end placed on a stand close to automatic sensors. Next we tested how the situation would change with the drone left at the same place, but this time hanging. The evaluation proved some variation, but that could be explained by spatial and time variability, that is predictable in an urban environment with building structures and inhomogeneous surface. Consequently we started parallel testing in flight and gradually terminated testing in stillness.

The comparison of temperature and relative humidity during flight was performed on three locations - airport Brno Medlánky, military training area Libavá and sounding station Prostějov. The testing focused on the different placement of sensors. When the measured data could not be compared to the real data and the tests were only limited to repetitiveness of value retrieval during different flight conditions, the tests pursued the accord between measurements taken during vertical ascent and descent with varying vertical speeds. Since there is usually no significant change of meteorological conditions in the course of minutes, the values obtained at the same position should correspond.

The first measurement was realized in training military area Libavá in April 2017. Different courses of flight were tested (see Fig. 2) and a height of approximately $850 \mathrm{~m}$ AGL was reached. The results could be summarized as follows:

1. The UAV Robodrone SuperHorned operated without problem and reached desired levels.

2. A great inertia of temperature sensor which caused difference between up and down measurement about $5 \mathrm{~K}$ (in low levels).

3. Big errors in relative humidity occurred, mainly caused by bad calibration constants.

4. Software and communication problems (in data downloading) resulted in a significant waste of time.

5. Outliers in pressure were detected. 


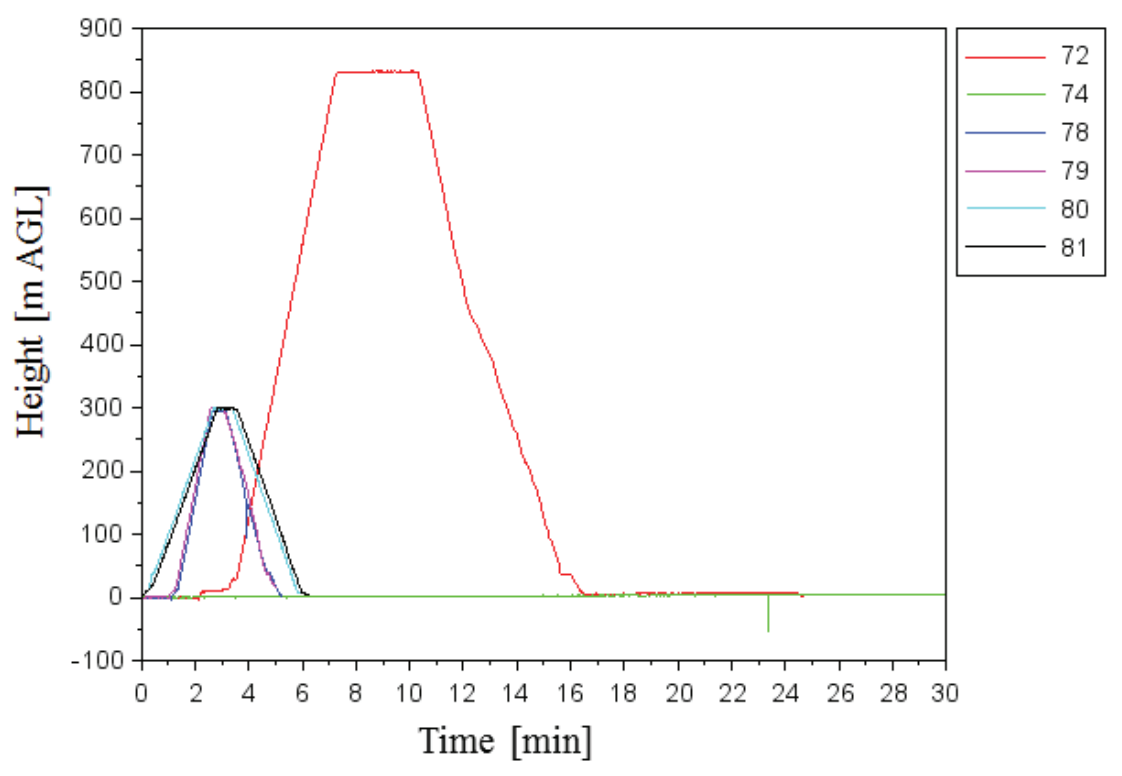

Fig. 2. Different course of flight tested in military training area Libavá

The above mentioned problems was figured out in cooperation with Robodrone Company (exchange and calibration of sensors, software improvement). For a more precise comparison, however, it is necessary to perform the comparison with real data. This took place in Prostějov, where the drone and meteorological probe with sensors by company Vaisala placed on a balloon were made to take off simultaneously (see Fig. 3). Another advantage of this measurement was the fact, that an identical probe to the one placed on the balloon could have been placed on the drone too. A complication to such comparison though is constituted in the raw-data processing from Vaisala in software on a processing device. A limitation of the comparison with upper-air sounding is the interval: only twice a day (every 12 hours), while utilization of supplementary probes is financially very demanding.

The described comparison method was applied in two days. The radio probe was placed on different parts of the drone (upper part, bottom part, bottom hanging part), so that the influence of drone itself on the temperature and humidity could be tested. The aforementioned fact, that raw data from probe are further computed and we do not know the computing algorithm, presented a limitation. It is questionable which characteristics apply to it and which conditions must be fulfilled in order to be able to use it and whether we actually even comply with them. Moreover, when pressure elevated, the software interprets it as probe falling and no calculation is done. The comparison of raw data and outgoing computed data usually returned temperature differences of less than $0.3 \mathrm{~K}$.

In the course of tests several modifications were made: adjustment of software for drone data processing (e.g. filter setup), clearing of discrepancies between pressure and temperature. For second round of testing another temperature sensor was added, so that there are two sensors available now - one up, second down. Another problem occurred with time synchronization that is necessary to carry out ahead of every start.

An illustration of temperature measurement comparison is on Fig. 4. V and Vsond present the same type of data from same equipment, yet several hundreds of meters above ground the difference amounts to $0,5 \mathrm{~K}$. Data from ascending part of flight of Vdron approximate the values of V and Vsond. Surprising is also the relatively notable difference in raw data from hanging probe at ascent and descent in upper part of ascent and a rather good concord in bottom part (computed data from descent are not available).

The testing of temperature measurement returned altogether good and optimistic results. One of the things the testing showed is variable offset, which would require calibration before every flight. Sensor inertia might still cause some problems and it is necessary to make more tests bearing this in mind. The thermal stratification on testing days was almost indifferent and it would be good to make similar tests also under more complex thermal stratification.

The issue of relative humidity measurement is more complicated. Values between ascent and descent differ significantly and even after a relatively long (several minutes) rest at standstill after flight the values do not get back to those before flight. On the other hand the humidity during flight does not vary much and the difference is usually less than $5 \%$. The comparison indicates that it is not realistic to expect determination of humidity with precision of less than $2 \%$. It is also opportune to test which values would be collected at flight through clouds and how would the humidifying manifest. 


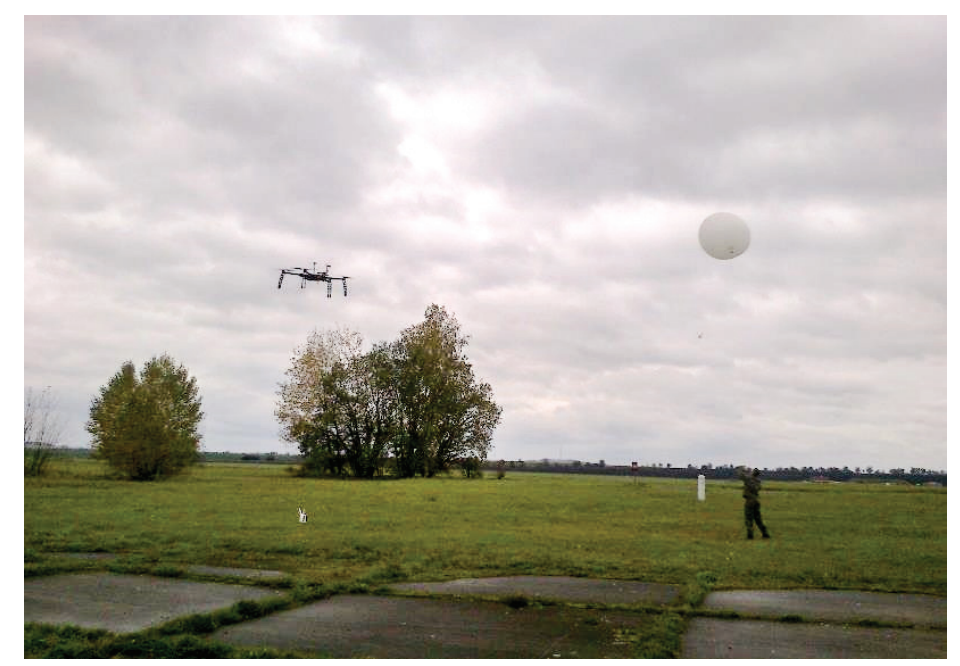

Fig. 3. Comparison of measurement with meteorological probe in Prostejov

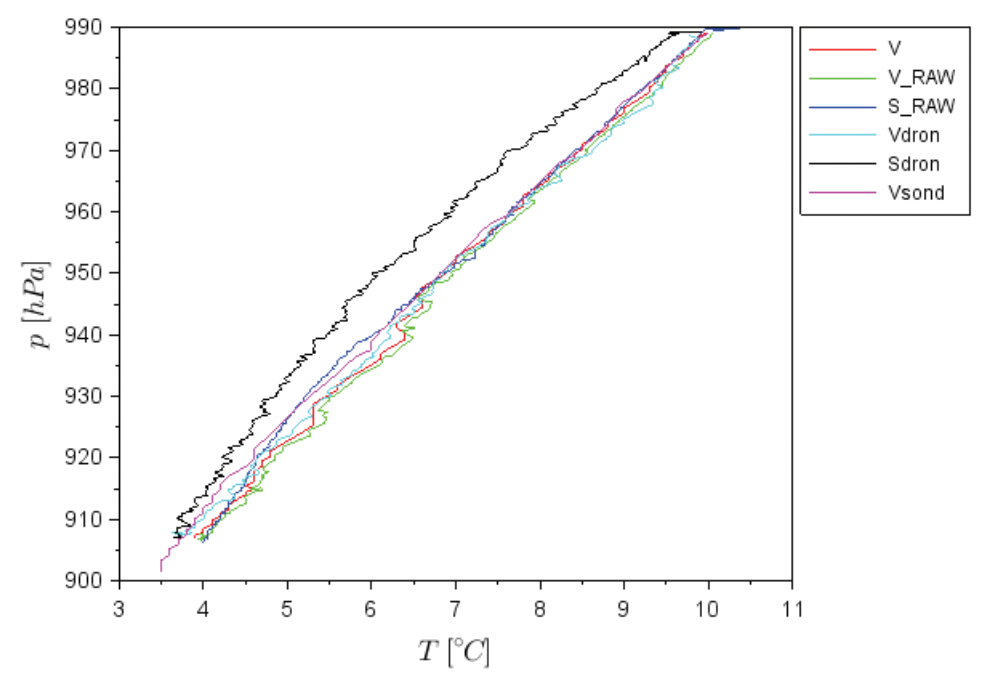

Fig. 4. Comparison of temperature measurement in Prostejov (27 October 2017 11:32 UTC). V - meteorological probe on $3 \mathrm{~m}$ the rope, V_RAW - raw data from this probe in ascending part, S_RAW - raw data from this probe in descending part, Vdron - dron (ascent), Sdron - dron (descent), Vsond - reguar meteorological probe (ballon)

Another possibility for comparison would be to use a $250 \mathrm{~m}$ high meteorological pole in Křešín (Pacov area), on which the sensors are positioned by every circa 50 meters. This distance is very rough, but the pole gives an advantage, that during one day several comparison measurements may be carried out. This possibility is though still in negotiation.

Unlike the meteorological measurement, obtaining the speed and direction of the wind is much more complicated. It is a process that requires to not only measure necessary input values but also quite a significant amount of aero-dynamical and mathematical apparatus. There are several possibilities to measure the parameters of the wind but all of them except one require to add more equipment which means that the drone must carry more weight and the precious flight time decreases. To fly to the $1500 \mathrm{~m}$ AGL that we decided to be our goal, and back already takes approximately 10 minutes assuming that the vertical speed is kept at $5 \mathrm{~m} / \mathrm{s}$. We therefore opted for a method that uses instrument that are already present on board of the drone.

Let assume that the drone is stationary in flight and wind is pushing the drone from this position. On board GPS system tries to correct this drift by tilting the drone slightly against the wind. The tilt is proportional to the wind speed and has an opposite orientation. The direction of the tilt can be calculated using the trigonometry and the wind direction can be determined. Adding the actual angle of the yaw and subtracting $180^{\circ}$ for the opposite orientation gives us the wind direction, see Fig. 5.Similarly, the speed of wind can be calculated but the mathematical decomposition is completely different. The speed can be derived from the forces acting on the drone while flying. In case the drone is stationary, the gravitational force is compensated by the thrust created by the four motors. Since we already calculated the tilt caused by the wind it is easy to determine the horizontal component of the thrust, which is closely connected with the wind speed, assuming the thrust and aerodynamic coefficients (drag) are known. 


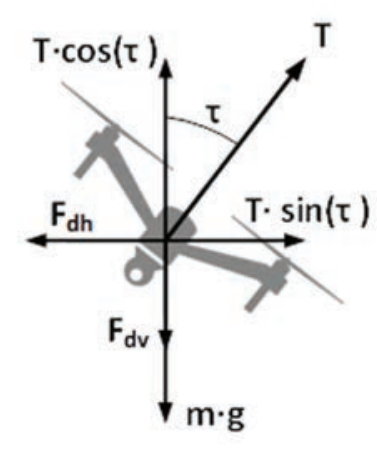

Fig. 5. Forces acting on the drone

Unfortunately, it is impossible for us at this moment to design an experiment in the aerodynamic tunnel to quantify the reference area and the drag coefficient. We therefore designed an experiment consisting of comparing known speed obtained from meteorological wind speed measurement device while hovering in close proximity. This experiment gave us a close approximation of the product of reference area and the drag coefficient. With this approximation second experiment has been done.

This time the experiment consisted of parallel flight of the drone and the meteorological balloon. This way we can compare data from both systems, see Fig. 6.
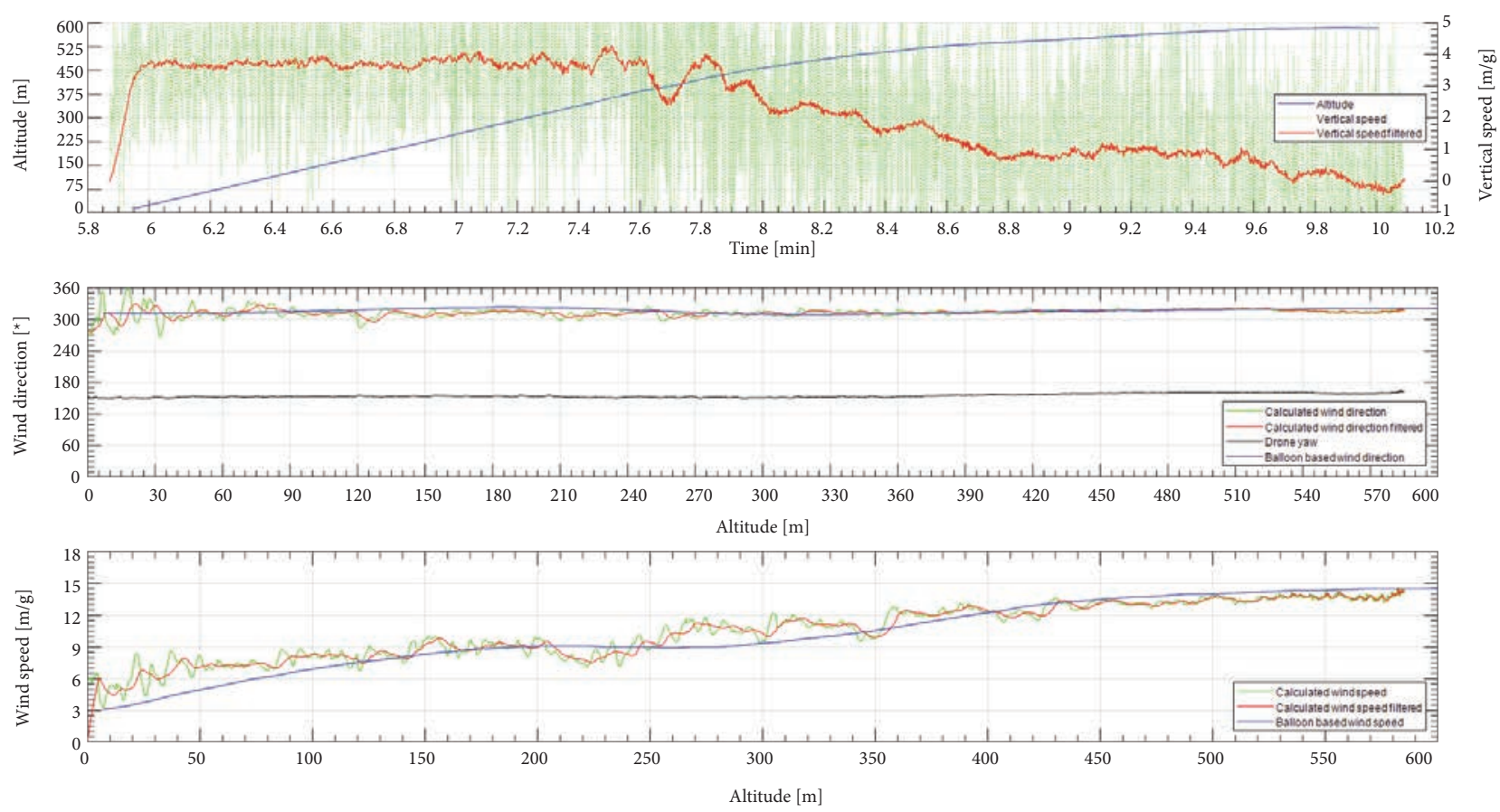

Fig. 6. Comparison between wind speed obtained from the balloon and estimated on drone

Those results show that there is a correlation between the wind speed measured by the balloon and estimated on the drone. They also show that the dynamic behavior of the drone influences the estimated speed and direction quite significantly. Balloon gives the measurements with a frequency of $0.5 \mathrm{~Hz}$ whilst the drone with a frequency of $10 \mathrm{~Hz}$. Another thing that must be mentioned is the fact that the balloon climbs at a rate of approximately 7-8 m/s and the drone with only 4-5 m depending on the wind strength and stability and that the balloon drifts away from the place where drone climbs. It means that the wind situation may be different. Finally, without the proper measurement from the wind tunnel, it is almost impossible to calculate the vertical component of force caused by the climbing movement and it was for this experiment consciously ignored.

\section{Conclusions}

The development and comparison brought about the following results. The selected temperature sensors have a considerably lower inertia than the ones originally tested. Instead of one, there are two sensors installed on the drone (one up, the other down), so that the varying influence of the drone on the temperature measurement during ascent and descent is eliminated. The development phase also exposed some software problems, which contributed to an incorrect 
determination of flight height. It appears (not only in comparison with Vaisala probe), that in order to get the real data with requested precision the collected data will have to undergo a post-processing. Another problem encountered during temperature measurement is a variable offset. Similarly, to the case of Vaisala probes, a calibration / temperature levelling will be necessary to do before the flight so that the corresponding values may be collected.

The testing to fix the most crucial problems. In order to verify and potentially correct the measured data it is necessary to carry out further comparative measurements at different meteorological conditions.

The direction and speed of wind can be calculated from UAV tilt because wind is pushing the hovering UAV from its position and GPS onboard system corrects this drift by tilting the UAV to redirect its thrust horizontal (lateral) component against the wind. Experimental results of the drone hovering and climbing in moderate and fresh breeze are presented including issues of determining the multicopter reference area and the drag coefficient as well as a difficulty to execute a valuable comparative measurement using a meteorological balloon.

\section{Acknowledgements}

The work presented in this paper was supported within the institutional support for "Development of the methods of evaluation of environment in relation to defense and protection of the Czech Republic territory" (NATURENVIR) by the Ministry of Defense of the Czech Republic.

\section{References}

1. Mayer S, Sandvik A, Jonassen MO, Reuder J. Atmospheric profiling with the UAS SUMO: A new perspective for the evaluation of fine-scale atmospheric models. Meteorol Atmos Phys 2012, 116 (1-2), p.15-26. ISSN: 01777971.

2. Rybanský, M., Břeňová, M., Čermák, J., Van Genderen, J., Sivertun, A. Vegetation structure determination using LIDAR data and the forest growth parameters. In: 8th IGRSM International Conference and Exhibition on Geospatial and Remote Sensing, IGRSM 2016. Kuala Lumpur, Malaysia: IOP Publishing Ltd, Dirac House, Temple Back, Bristol BS1 6BE, England, 2016. ISSN 1755-1307.

3. Reuder J, Brisset P, Jonassen M, Müller M, Mayer S. The Small Unmanned Meteorological Observer SUMO: A new tool for atmospheric boundary layer research. Meteorol Z, 2009, 18 (2), p.141-147. ISSN: 09412948.

4. Reuder J, Jonassen MO, Ólafsson H. The Small Unmanned Meteorological Observer SUMO: Recent developments and applications of a micro-UAS for atmospheric boundary layer research. Acta Geophys 2012, 60 (5), p.1454-1473. ISSN 1895-7455.

5. Hemingway BL, Frazier AE, Elbing BR, Jacob JD. Vertical sampling scales for atmospheric boundary layer measurements from small unmanned aircraft systems (sUAS). Atmosphere, 2017, 8 (9). ISSN: 20734433.

6. Dejmal K, Hudec F, Kolář P, Novotný J. Evaluation of measurement quality of selected elements on the meteorological stations Meteos6 and Davis Vantage Pro 2 in the military quarters area of Černá Pole. In: Conference Proceedings of ICMT'17. Piscataway, NJ 08854-4141 USA: Institute of Electrical and Electronics Engineers Inc., 2017, p.318-324. ISBN 978-1-5386-1988-9.

7. Dejmal K, Almášiová L. The comparison of temperature and moisture characteristics of natural and artificial surfaces. In: Central Europe Area in View of Current Geography. Proceedings of 23rd Central European Conference. Brno: Masarykova univerzita, 2016, p. 141-148. ISBN 978-80-210-8313-4.

8. Bystřický R, Novotný J, Dejmal K. Use of the climatic chamber for meteorological drone validation. In: 17. International Scientific Conference „Measurement, Diagnostics and Dependability of Aircraft Systems 2017“. Brno: University of Defence, 2017, p. 50-59. ISBN 978-80-7582-012-9.

9. Robodrone - Superhornet. [Online], Available at: https://www.robodrone.com/en/superhornet 